gene (1989). Analysis of the gene led to definition of the protein CF transmembrane receptor which turned out to be the chloride channel. International CF meetings now attracted thousands and there was riotous delight as Francis Collins, one of the team leaders, announced the identification of the CF gene with a song he had written accompanied by a small guitar.

\section{New treatments}

New treatments followed, not always because of the new science but rather because of a concerted effort by clinicians, CF groups and small pharmaceutical companies - although rare, life-long treatment of CF could provide reasonable returns and new therapies might be extended to other more common airway diseases. First came deoxyribonuclease (DNase), a recombinant human protein that reduced sputum viscosity and improved lung function. Then came Tobi ${ }^{\circledR}$, a new nebulisable formulation of tobramycin. Nebulised antibiotics had been used for 20 years in Europe but it needed a link up between the CF Foundation and a small pharmaceutical company to fund a series of definitive clinical trials. At the same time major progress in lung transplantation restored a few to normal life and gave hope to many more.

\section{New devices}

Alongside all of this, and just as important, were the new devices. Nasogastric feeding tubes were replaced by small bore percutaneous endoscopic gastrostomy tubes - better and kinder. Permanent iv access by subcutaneous ports made iv antibiotic treatment easier. Positive pressure iv infusion devices allowed treatment to continue at home or at work and improved topical lung delivery systems - rapid nebulisers and dry powder inhalers simplified inhaled treatment and enhanced compliance. Positive pressure and oscillatory devices assisted physiotherapy and noninvasive ventilators palliated symptoms for advanced lung disease and bridged a few to transplantation.

All these technical advances coincided with and contributed to a revolution in the delivery of care. Cystic fibrosis centres built up multidisciplinary teams made up of specialist nurses, physiotherapists, dieticians, pharmacists and microbiologists. National and international guidelines defined standards of staffing, monitoring, treatment and cross infection. Cystic fibrosis databases added to knowledge of epidemiology and allowed comparisons between individual clinics and different countries so providing evidence of optimal care. The CF community pioneered patient advocates, expert patients, peer review of CF centres, and the CF organisations became formidable political lobbyists, fundraisers and directors of research.

\section{New problems}

All this energy, progress and enthusiasm have improved the lives of people with CF far beyond the expectations of the 1960s but inevitably new problems have been identified:
- longer life means more time to develop complications as diabetes, osteoporosis, sexual and psychological difficulties are now a routine part of CF care

- cross infection has plagued a few centres and new antibiotic resistant pathogens are becoming an increasing problem

- costs of care are escalating

- the intensity of treatment is becoming more burdensome and the risks of long-term side effects are steadily increasing.

All these are challenges for the future to be set against the gains that are now so visible. Now CF is no longer a miserable, fatal childhood disease but instead is a lifelong medical condition with an estimated prognosis of at least 40 years and rising. Those with CF are able to work normally and raise families albeit with a major burden of treatment and uncertainty about the future. A massive and well coordinated research effort is under way. High throughput screening of tens of thousands of chemical entities should find new ion transport drugs and gene therapy (proof of principle already published) is developing steadily. Further progress will follow. Cystic fibrosis as a lifelong condition will remain but $\mathrm{CF}$ as a disease is on its way out.

\section{Genetic predisposition to chronic obstructive pulmonary disease: advances in $\alpha_{1}$-antitrypsin deficiency and the serpinopathies}

David A Lomas PhD ScD FRCP FMedSci

Professor of Respiratory Biology; Honorary Consultant Physician; Deputy Director, University of Cambridge, Cambridge Institute for Medical Research

Email: dal16@cam.ac.uk

Alpha ${ }_{1}$-antitrypsin deficiency is the only widely accepted genetic factor that predisposes smokers to chronic obstructive pulmonary disease (COPD). Most individuals with COPD have normal levels of $\alpha_{1}$-antitrypsin but $1-2 \%$ are homozygous for the severe $Z$ deficiency allele (Glu342Lys). This mutation causes $\alpha_{1}$-antitrypsin to be retained within the endoplasmic reticulum of hepatocytes as periodic acid Schiff (PAS) positive inclusions. Work over the past 15 years has elucidated the mechanism by which the mutant protein is retained within the liver and has provided new insights into the pathobiology of the liver and lung disease associated with $\alpha_{1}$-antitrypsin deficiency. Biochemical, biophysical and crystallographic studies have shown that the $Z$ mutation of $\alpha_{1}$-antitrypsin causes the protein to undergo an aberrant conformational transition and form chains of ordered polymers. ${ }^{1-3}$ These ordered polymers are not detected by the unfolded protein response ${ }^{4}$ and are only partly cleared by autophagy. Their retention within hepatocytes causes cell damage that presents clinically as neonatal 
hepatitis, cirrhosis and hepatocellular carcinoma. ${ }^{5}$ It has become clear that this process also underlies the deficiency of other mutants of $\alpha_{1}$-antitrypsin. The Siiyama mutation (Ser53Phe) is the most common cause of $\alpha_{1}$-antitrypsin deficiency in Japan and the Mmalton ( $\Delta 52$ Phe) variant is the most common cause of $\alpha_{1}$-antitrypsin deficiency in Sardinia. Both mutations similarly favour the formation of polymers that are retained within hepatocytes as PAS positive inclusions. ${ }^{3}$ Polymerisation also explains the mild deficiency of S (Glu264Val) and I (Arg39Cys) $\alpha_{1}$-antitrypsin. In each case there is a striking genotype/phenotype correlation that can be explained on the basis of polymerisation. Those mutants that cause the most rapid polymerisation cause the most severe retention of protein within hepatocytes, the most profound plasma deficiency and the greatest risk of liver disease.

\section{Proteinase inhibitors in the lung}

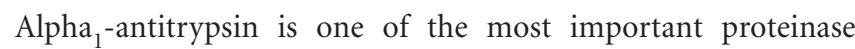
inhibitors within the lung. Emphysema associated with $\alpha_{1}$-antitrypsin deficiency is widely believed to be due to uncontrolled action of the enzyme neutrophil elastase giving rise to tissue destruction. The situation is exacerbated as the $\mathrm{Z}$ mutation reduces the association rate between $\alpha_{1}$-antitrypsin and neutrophil elastase by approximately fivefold. The inhibitory activity of $\mathrm{Z} \alpha_{1}$-antitrypsin can be further reduced as the $\mathrm{Z}$ mutation favours the spontaneous formation of $\alpha_{1}$-antitrypsin loop-sheet polymers within the lung. ${ }^{6}$ This conformational transition inactivates $\alpha_{1}$-antitrypsin as a proteinase inhibitor, thereby further reducing the already depleted levels of $\alpha_{1}$-antitrypsin that are available to protect the alveoli. Moreover the conversion of $\alpha_{1}$ antitrypsin from a monomer to a polymer converts it to a chemoattractant for human neutrophils. ${ }^{7,8}$

The magnitude of the effect is similar to that of the chemoattractant C5a and present over a range of physiological concentrations. More recently a monoclonal antibody has been used to demonstrate the co-localisation of polymers of $\alpha_{1}$-antitrypsin and neutrophils in emphysema associated with $\mathrm{Z} \alpha_{1}$-antitrypsin deficiency but not in emphysema in individuals with normal levels of $\alpha_{1}$-antitrypsin. ${ }^{9}$ Therefore the chemoattractant properties of polymers may combine with tobacco smoke to drive the progressive emphysema seen in individuals with $\alpha_{1}$-antitrypsin deficiency.

\section{Serpinopathies}

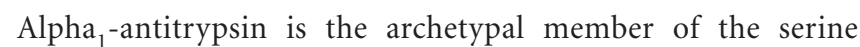
proteinase inhibitor (or serpin) superfamily of proteins. They are all linked by a common molecular structure but they inhibit different target enzymes. The process of polymerisation also occurs spontaneously in association with mutants of other members of this family to cause intracellular hepatic retention and plasma deficiency. This results in deficiency of antithrombin, C1-inhibitor and $\alpha_{1}$-antichymotrypsin that has been associated with thrombosis, angiooedema and COPD respectively. It also results in the plasma deficiency of heparin co-factor II but this has yet to be associated with a clinical phenotype. The process is perhaps most strikingly displayed in mutants of a neurone specific serpin, neuroserpin, to cause PAS positive intracerebral polymers that underlie the dementia familial encephalopathy with neuroserpin inclusion bodies or FENIB. ${ }^{10}$ In view of the common molecular mechanism, these conditions have been grouped together as a new class of disease that has been called the serpinopathies. ${ }^{3}$ The new insights provided by understanding serpin polymerisation has allowed the development of novel therapeutic strategies to block the aberrant conformational transition and so treat the associated disease. ${ }^{3}$

\section{References}

1 Lomas DA, Evans DL, Finch JT, Carrell RW. The mechanism of $\mathrm{Z} \alpha_{1}$-antitrypsin accumulation in the liver. Nature 1992;357:605-7.

$2 \mathrm{Yu} \mathrm{M-H,} \mathrm{Lee} \mathrm{KN,} \mathrm{Kim} \mathrm{J.} \mathrm{The} \mathrm{Z} \mathrm{type} \mathrm{variation} \mathrm{of} \mathrm{human} \alpha_{1}$-antitrypsin causes a protein folding defect. Nat Struc Biol 1995;2:363-7.

3 Lomas DA, Mahadeva R. Alpha-1-antitrypsin polymerisation and the serpinopathies: pathobiology and prospects for therapy. J Clin Invest 2002;110:1585-90.

4 Hidvegi T, Schmidt BZ, Hale P, Perlmutter DH. Accumulation of mutant alpha $\mathrm{1}_{1}$-antitrypsin $\mathrm{Z}$ in the endoplasmic reticulum activates caspases-4 and -12, NFkappaB, and BAP31 but not the unfolded protein response. J Biol Chem 2005;280:39002-15.

5 Sveger $\mathrm{T}$. The natural history of liver disease in $\alpha_{1}$-antitrypsin deficient children. Acta Paed Scand 1988;77:847-51.

6 Elliott PR, Bilton D, Lomas DA. Lung polymers in $\mathrm{Z} \alpha_{1}$-antitrypsin related emphysema. Am J Respir Cell Mol Biol 1998;18:670-4.

7 Parmar JS, Mahadeva R, Reed BJ et al. Polymers of $\alpha_{1}$-antitrypsin are chemotactic for human neutrophils: a new paradigm for the pathogenesis of emphysema. Am J Respir Cell Mol Biol 2002;26: 723-30.

8 Mulgrew AT, Taggart CC, Lawless MW et al. $\mathrm{Z} \alpha_{1}$-antitrypsin polymerizes in the lung and acts as a neutrophil chemoattractant. Chest 2004;25:1952-7.

9 Mahadeva R, Atkinson C, Li J et al. Polymers of Z $\alpha_{1}$-antitrypsin co-localise with neutrophils in emphysematous alveoli and are chemotactic in vivo. Am J Pathol 2005;166:377-86.

10 Davis RL, Shrimpton AE, Holohan PD et al. Familial dementia caused by polymerisation of mutant neuroserpin. Nature 1999;401:376-9.

\section{Management of rare diseases in respiratory medicine}

\author{
Simon R Johnson BSc DM MRCP \\ Reader in Respiratory Medicine, University Hospital, \\ Queens Medical Centre, Nottingham
}

Email: simon.johnson@nottingham.ac.uk

General medicine comprises caring for patients with a fairly small number of common conditions and a much larger range of rare conditions. Medicine in any specialty will involve seeing patients with rare diseases. As a whole, rare diseases affect large numbers of patients, with up to 25 million Americans affected by 6,000 different conditions. ${ }^{1}$ Diagnosis and optimal treatment is part of all physicians workload but such patients pose a series of challenges. Firstly, delays, sometimes of several years, in the diagnosis of rare conditions can occur when the symptoms, although 